\title{
Múltiplos olhares sobre a produção do conhecimento em educação especial
}

\author{
Multiple views on the production of knowledge \\ in special education
}

\section{Maria Cristina Piumbato Innocentini Hayashi}

Professora associada do Departamento de Ciência da Informação e do Programa de Pós-Graduação em Educação Especial da Universidade Federal de São Carlos (UFSCar), São Carlos, SP, Brasil, e-mail: dmch@ufscar.br

\section{Resumo}

O artigo aborda a produção do conhecimento em educação especial enfocando aspectos como as abordagens e vertentes epistemológicas da área, o atendimento de demandas sociais em educação especial promovido pelas pesquisas realizadas, a construção teórica, os procedimentos metodológicos e a efetividade dos resultados das pesquisas empreendidas neste campo de conhecimento. Ao mesmo tempo, problematiza questões a respeito de como o conhecimento produzido na universidade pode ter impacto na prática da educação inclusiva fora dos muros acadêmicos e como as pesquisas podem transformar a realidade da educação especial no Brasil de hoje. Finaliza com uma reflexão sobre os incentivos públicos ou privados de fomento à produção do conhecimento em educação especial no Brasil.

Palavras-chave: Educação especial. Pesquisa e produção de conhecimento. Produção científica. 


\begin{abstract}
The article discusses the production of knowledge in special education focusing on approaches and epistemological aspects of the area, the attention to social demands in special education sponsored by the research, the theoretical construct, methodological procedures and the effectiveness of research results in this field of knowledge. At the same time raises questions about how the knowledge produced in university may have an impact on the practice of inclusive education outside the walls of academia and how research can transform the reality of special education in Brazil today. Concludes with a discussion of the public or private incentives to encourage the production of knowledge in special education in Brazil.
\end{abstract}

Keywords: Special education. Research and knowledge production. Scientific production.

\title{
Introdução
}

A abrangência do campo da educação especial e suas interfaces com outras áreas do conhecimento refletem-se na diversificada produção científica da área, que se encontra consolidada em periódicos científicos nacionais e internacionais, indexados em bases de dados. Em virtude do caráter multidisciplinar do próprio campo da educação especial, as pesquisas desenvolvidas em seu interior incorporam métodos e técnicas de outras áreas, as quais permitem refletir sobre aspectos relacionados à prevenção, à reabilitação e ao desenvolvimento das habilidades das pessoas com deficiência, ${ }^{1}$ conforme já assinalado por Mazzotta (1996).

1 Ao longo da história da educação especial, várias terminologias foram utilizadas para designar as pessoas com necessidades especiais: portadores de necessidades especiais, portadores de deficiência, deficientes, pessoas com deficiência, entre outras. Entre esses termos, "pessoa com deficiência" parece ser o mais adequado, uma vez que a deficiência é uma condição e não algo para ser portado. Apesar disso, neste texto não adotamos uma terminologia única, pois o foco do artigo é a produção científica sobre as pessoas com deficiência e, nesse caso, a terminologia varia conforme a visão do(s) autor(es). No entanto, concordamos com Sassaki (2003, p. 160) que "a construção de uma verdadeira sociedade inclusiva passa também pelo cuidado com a linguagem"

Rev. Diálogo Educ., Curitiba, v. 11, n. 32, p. 145-165, jan./abr. 2011 
Desde os anos 1980, quando pesquisadores desse campo começaram a avaliar os estudos produzidos até os dias atuais, várias pesquisas foram desenvolvidas na perspectiva de avaliar o conhecimento produzido pela área. Neste artigo pretende-se traçar um panorama desses estudos por meio da problematização de algumas questões relacionadas ao estado do conhecimento na área de educação especial na atualidade e sobre os impactos dessa produção no contexto da universidade - locus privilegiado que congrega os pesquisadores do campo e produtores de conhecimento especializado - e da sociedade. O texto está organizado em seis partes, além da introdução e conclusão. Nos dois primeiros tópicos são enfocadas as abordagens epistemológicas adotadas nas pesquisas da área e as construções teóricas, procedimentos metodológicos e efetividade dos resultados das pesquisas realizadas. Os demais itens constituem-se em uma tentativa de responder a questões relacionadas às repercussões sociais das pesquisas na área e sobre os mecanismos de apoio público e privado disponíveis no País às pesquisas no campo da educação especial.

\section{Abordagens e vertentes epistemológicas das pesquisas em educação especial}

Um dos trabalhos de referência sobre a produção científica em educação especial no Brasil é o de Ferreira (1991), que examinou os catálogos da Anped, no período de 1981 a 1988, que continham os resumos das dissertações e teses defendidas nos Programas de Pós-Graduação em Educação no País. O autor verificou que, dos quase 1.900 trabalhos, 99 envolveram "alunos especiais", e que a maioria das pesquisas foi realizada a partir de 1984 e se encontrava vinculada às áreas de educação especial dos Programas de Pós-Graduação da UFSCar e da UERJ. Os trabalhos

e que esta "expressa, voluntariamente ou involuntariamente, o respeito ou a discriminação em relação às pessoas com deficiências". O mesmo se deu, no texto, com o termo "deficiência". O estudo de Mendes (1995) é referência para a discussão desse conceito. 
contemplavam as diferentes áreas de "excepcionalidade", porém enfatizavam a deficiência mental, os procedimentos de instrução e as condições de funcionamento das classes especiais.

Em 1995, Toresan, Reily e Caiado analisaram dissertações e teses defendidas em universidades paulistanas cujos temas tinham relação com a deficiência e verificaram que a maioria dos estudos foi produzida na USP, além de estarem inseridos na área médica e direcionados para a deficiência mental. Em 1996, Nunes Sobrinho chama a atenção para alguns fatores presentes nos estudos na área de educação especial: número limitado das teorias que embasam os estudos na área; resolução de apenas problemas imediatos; necessidade de investigações sobre identificação, triagem e encaminhamento para serviços especiais; necessidade de pesquisas interdisciplinares sobre prevenção de acidentes na infância; identificação de variáveis que interferem no desempenho de professores nas séries iniciais.

Por sua vez, Almeida e Marquezine (1997) analisaram 77 monografias de um curso de especialização em Educação Especial em Deficiência Mental no período de 1987 a 1997 e verificaram que a maioria dos estudos referia-se a pesquisas sobre intervenção, seguidos por levantamentos, estudos de caso, revisões de literatura e pesquisa histórica. As temáticas pesquisadas nos estudos foram: "cognição e leitura escrita", "Educação Física”, "desempenho de professores e técnicos na escola", "avaliação da pessoa deficiente”, atitudes e participação da família, capacitação dos não profissionais.

Em relação às temáticas da educação especial, são múltiplas as abordagens e vertentes epistemológicas comumente encontradas nos trabalhos desenvolvidos pelos pesquisadores. Se realizarmos um estado da arte sobre avaliação da produção científica em educação especial, com o objetivo de sistematizar e analisar a literatura da área, os resultados apontarão que embora existam vários estudos, são poucos aqueles que se dedicam a analisar as vertentes epistemológicas das pesquisas realizadas. Algumas exceções devem ser mencionadas:

1) O projeto Prodisc - desenvolvido no fim dos anos 1990 até a primeira metade dos anos 2000, por um grupo de pesquisadores 
da UFSCar, UERJ e Unimep - analisou a produção científica representada pelas teses e dissertações com interface na educação especial oriundas dos programas de Pós-Graduação em Educação e Psicologia, desde 1970 até 2004. Esse projeto teve quatro fases, e Sacardo (2006, p. 28) sintetizou-as da seguinte maneira: na primeira etapa, em 1995, os autores analisaram a produção científica discente do PPGEEs/UFSCar e do PROPEd/ UERJ e constataram que as pesquisas realizadas frequentemente não se referiam aos aspectos históricos da educação especial, além de não relacionarem o problema investigado ao contexto no qual se inseria a educação no País. Na segunda etapa, os autores analisaram 98 teses e dissertações defendidas em 22 programas de Pós-Graduação em Educação no Brasil, cujas temáticas incidiram sobre as pessoas com deficiência, e verificaram a predominância de estudos sobre a perspectiva social sobre a psicoeducacional. Na terceira etapa do Prodisc, a produção científica discente do período 1996 a 1999 foi analisada e os resultados revelaram proximidade crescente dos referenciais e problemas presentes na educação regular. Finalmente, as análises críticas da quarta fase do Prodisc revelaram que a produção científica discente tinha como temáticas principais a educação das pessoas com deficiência, os distúrbios de conduta e os talentos especiais.

Em 2004, Nunes, Ferreira \& Mendes analisaram a produção científica da área de educação especial tomando como objeto de estudo a produção discente oriunda de programas de pós-graduação e linhas de pesquisa nas áreas de educação e psicologia. Os resultados indicaram que o locus dessa produção é as universidades públicas e que os estudos oriundos da área da educação são predominantes. Além disso, verificaram que o tema da deficiência mental é preponderante em relação a outras categorias de deficiências, que os estudos realizados ocorreram, em sua maioria, em ambiente escolar, e que a faixa etária preferencial 
compreendeu crianças de 7 a 12 anos. Do ponto de vista metodológico, os autores identificaram a predominância das pesquisas descritivas nos estudos das duas áreas, com maior destaque na área da educação (56\% dissertações e teses) do que na psicologia ( $43 \%$ dissertações e teses). Os autores também verificaram que o segundo tipo de delineamento mais frequente foi o experimental na psicologia (17\%) e o estudo de caso na educação (21\%) e na psicologia (17\%); a concepção de deficiência/diferença manifesta nos trabalhos caracteriza-se como psicoeducacional (NUNES; FERREIRA; MENDES, 2004)

2) Em 2004, Silva, em sua dissertação de mestrado defendida no Programa de Pós-Graduação em Educação Especial da UFSCar, realizou uma análise epistemológica de 27 dissertações e teses do PPGEEs/UFSCar no período 1981 de 2002 e verificou que a maioria dos estudos adotou a abordagem empírico-analítica, seguida em menor número pela abordagem fenomenológico-hermenêutica. Além disso, constatou que nenhum estudo utilizou a abordagem crítico-dialética. O estudo também apontou que $44,4 \%$ das dissertações e teses caracterizaram-se como pesquisa descritiva, $40,7 \%$ utilizaram o delineamento experimental, 7,4\% foram pesquisas bibliográficas e $7,4 \%$ não especificaram o delineamento.

3) Mais recentemente, em 2006, em outro estudo de análise da produção científica em educação especial, Laplane, Lacerda e Kassar (2006) verificaram que o leque das pesquisas em educação especial foi ampliado nas últimas décadas, seja pela escolha de diferentes temáticas (relações de ensino; gênero; identidade; relações com o conhecimento; formação de professores; política educacional; cultura e arte), seja pela diversificação de enfoques teórico-metodológicos (psicologia social; teoria da aprendizagem mediada; história oral; análise de discurso; história e sociologia). As autoras desse estudo assinalaram que parte das pesquisas que privilegiam as relações do sujeito com seu meio social se fundamenta na psicologia histórico-cultural, a partir dos aportes teóricos do 
materialismo histórico, incorporando a noção de que o sujeito se desenvolve imerso nas relações sociais. Essa concepção tem implicações para o tipo de pesquisa empreendida e a educação especial passa a ser compreendida como foco de interesse dentro do contexto da educação em geral. As autoras concluem que tal abordagem tem produzido trabalhos que enfocam aspectos micro e macro constitutivos da participação de sujeitos nas práticas sociais.

4) Em 2008, cinco pesquisadores da Universidade Federal de Juiz de Fora (Marques, Carneiro, Andrade, Martins, Gonçalves) estudaram a questão da articulação lógica entre o problema e a proposição teórico-metodológica das pesquisas na área da educação especial, tendo como foco os seus pressupostos epistemológicos. O corpus da pesquisa foi constituído de 85 teses e dissertações defendidas entre 2001 e 2003 em 24 Programas de Pós-Graduação em Educação e em Educação Especial do Brasil, disponíveis no banco de teses da CAPES. Os autores utilizaram na análise dos trabalhos a matriz paradigmática de Sanchez Gamboa (1998), que considera os pressupostos teóricos, metodológicos, lógico-epistemológicos, gnosiológicos, ontológicos e analíticos das pesquisas. Os resultados obtidos apontaram que a tendência epistemológica presente em $67 \%$ dos trabalhos era a fenomenológico-hermenêutica, seguidas pela empírico-analítica (22\%) e pela crítico-dialética (11\%).

5) Em 2009, Lourenço, Hayashi e Almeida conduziram estudo que visou a identificar e caracterizar as dissertações e teses com delineamentos intrassujeitos, produzidas desde a implementação do Programa de Pós-Graduação em Educação Especial (PPGEEs) da Universidade Federal de São Carlos (UFSCar), ocorrida em 1978, até o ano de 2004. Os resultados revelaram que os delineamentos intrassujeitos presentes nas dissertações e teses analisadas podem possibilitar suficiente demonstração do controle experimental e impacto das intervenções, e sua utilização em 
pesquisas sobre educação especial pode minimizar as lacunas no conhecimento dessa temática, além de permitir que se caminhe em direção à superação da distância entre pesquisa e prestação de serviços, já que se aplicam em escolas, clínicas, hospitais e lares e em áreas como educação, psicologia, educação especial, entre outras.

Assim, os estudos de análise da produção científica no campo da educação especial têm apontado diferentes perspectivas teóricas, conceituais e metodológicas, com relação aos objetos de estudo e abordagens, indicando aos pesquisadores da área que a diversidade de perspectivas propicia um diálogo entre as múltiplas formas de aprofundamento teóricoconceitual da área.

\section{Construção teórica, procedimentos metodológicos e efetividade dos resultados das pesquisas}

A construção teórica, os procedimentos metodológicos e a efetividade dos resultados das pesquisas realizadas no campo da educação especial são aspectos que demandam estudo aprofundado sobre a produção cientifica da área, buscando revelar a sua estrutura, o seu grau de abrangência e o seu nível de profundidade, à luz do estado da arte, o que não é tarefa simples.

Com base nas pesquisas sobre a produção científica nesse campo de conhecimento, pode-se supor que a qualidade do conhecimento produzido em educação especial em relação à criticidade, ao posicionamento assumido pelos autores, aos procedimentos metodológicos adotados e à efetividade dos resultados pode ser considerada de bom nível.

Um indicador dessa qualidade pode ser buscado nas pesquisas de metanálise do conhecimento científico produzido pela área. Esses estudos têm como objetivo realizar revisões críticas sobre essas produções, 
além de produzir indicadores a respeito da população-alvo enfocada nas pesquisas realizadas (faixa etária, sexo, escolaridade); as teorias que embasam esses estudos e a abordagem metodológica adotada; os tipos de estudos realizados (por exemplo, de intervenção, levantamento, revisões de literatura, pesquisa histórica); e a concepção de deficiência adotada (psicoeducacional, médico-clínica).

Outra vertente desses estudos é aquela que utiliza a abordagem bibliométrica para analisar a produção científica da área de educação especial (SILVA, 2004). Por meio de levantamento da produção científica consolidada em artigos científicos, teses e dissertações, livros e capítulos de livros, disponível em bancos de dados e bibliotecas digitais e em periódicos científicos indexados em bases de dados nacionais e internacionais, essas pesquisas levam a própria área a refletir criticamente sobre os avanços e retrocessos do conhecimento produzido nesse campo. Por exemplo, o estudo já citado aqui e realizado pelos pesquisadores da Universidade Federal de Juiz de Fora (MARQUES et al., 2008) mostraram alguns equívocos das pesquisas da área de educação especial:

a não inserção da pesquisa entre as produções na área da Educação Especial e na Educação em geral; a ausência de criticidade em relação a outras teorias; o não posicionamento do autor ou da autora numa determinada concepção de educação; a construção teórica fundamentada em autores de concepções diferentes; a falta de coerência entre os pressupostos teóricos e metodológicos; a não explicitação da metodologia e dos procedimentos metodológicos adotados; a não descrição dos procedimentos utilizados para se garantir a ética na realização da pesquisa; a elaboração de conclusões que não apresentam relações com a análise feita; e a má elaboração dos resumos (MARQUES et al., 2008, p. 262-263).

São questões importantes, que mostram como a própria área tem condições de refletir sobre sua produção e buscar mecanismos para superar essas lacunas. 


\section{Quais demandas sociais as pesquisas acadêmicas em educação especial atendem?}

As pesquisas na área da educação especial abrangem uma amplitude de temáticas e têm contribuído não só para o avanço do conhecimento desse campo científico, como também têm produzido impactos em termos do desenvolvimento integral da pessoa com deficiência. Abarcam desde aspectos relacionados às atividades da vida cotidiana, passando pela sua interação com pais, familiares, educadores até questões relacionadas, por exemplo, com a legislação e os direitos. Em suma, essas pesquisas visam à interação social, ao desempenho e à qualidade de vida dessas pessoas, o que sinaliza o alto grau de comprometimento deste campo de conhecimento para com as demandas sociais e culturais postas pela sociedade.

Vários são os exemplos de pesquisas realizadas que podem ser citados aqui, uma vez que têm buscado atender demandas sociais e culturais das pessoas com deficiência, e por esse motivo têm alcançado grande impacto social. Nesse enquadramento, são dignas de serem mencionadas as pesquisas que objetivaram:

1) o desenvolvimento, a utilização e a avaliação de tecnologias assistivas, que buscam verificar como essas tecnologias podem promover o máximo de autonomia, independência, qualidade de vida e inclusão social das pessoas com deficiência. As tecnologias assistivas são constituídas por produtos, recursos, metodologias, estratégias, práticas e serviços que objetivam promover a funcionalidade, relacionada à atividade e participação, de pessoas com deficiência, incapacidades ou mobilidade reduzida e que abarcam uma série de possibilidades do desempenho humano, desde tarefas básicas de autocuidado (mobilidade, comunicação, manutenção do lar, preparo de alimentos, tarefas ocupacionais), até atividades de lazer e de trabalho. Estudos que têm como foco as tecnologias assistivas revelam novas perspectivas para a inclusão de pessoas com deficiência; 
2) a inserção da pessoa com deficiência no mercado de trabalho. Aqui, os estudos estão dirigidos para o processo de formação e qualificação profissional, bem como para compreender os fatores sociais, econômicos e individuais que dificultam a inserção dessas pessoas no mercado de trabalho. Essas pesquisas buscam ultrapassar os aspectos meramente relacionados, por exemplo, à legislação específica que normatiza o regime especial de trabalho protegido, e caminham, por exemplo, na direção de propor e avaliar o planejamento e a organização de serviços para a formação de pessoas com deficiência para o trabalho.

Outros exemplos, além desses, podem ser buscados na UFSCar, particularmente no Programa de Pós-Graduação em Educação Especial, único programa de pós-graduação strictu sensu do País nessa área, que se organiza em torno de cinco linhas de pesquisa que buscam produzir conhecimentos específicos para atender às demandas sociais e culturais das pessoas com deficiência, a saber:

1) Aprendizagem e cognição de indivíduos com necessidades especiais de ensino - que investiga processos básicos de aprendizagem e cognição e possíveis comprometimentos desses processos em indivíduos com necessidades especiais de ensino, incluindo deficiência mental, autismo ou problemas de aprendizagem.

2) Currículo funcional: implementação e avaliação de programas alternativos de ensino especial - desenvolve estudos para a identificação, descrição e superação de necessidades educativas especiais, propondo, implementando e avaliando cientificamente programas educacionais sistêmicos, considerando o ensino de habilidades específicas, a estruturação de rotinas pedagógicas, a proposição de parâmetros curriculares ou mesmo a análise de programas e serviços de ensino especial.

3) Práticas educativas: processos e problemas - estuda processos envolvidos nas práticas educativas, e no aperfeiçoamento dessas 
práticas, para a promoção do desenvolvimento e da aprendizagem de pessoas com necessidades educativas especiais.

4) Atenção primária e secundária em educação especial: prevenção de deficiências - focaliza a investigação de fatores de risco biológico e ambiental associados à prevenção e também ao desenvolvimento de programas de intervenção ou de aplicações a serviços nas áreas de educação e saúde. Dá ênfase ao estudo do processo de desenvolvimento humano e dá atenção específica ao diagnóstico de condições especiais (seja de risco ou de proteção) em qualquer etapa do ciclo vital e também ao aprimoramento de técnicas que minimizem os efeitos estressores dessas condições especiais. São estudados padrões de interação entre a família e o indivíduo em situação de risco ou a pessoa com deficiência.

5) Produção científica e formação de recursos humanos em Educação Especial - focaliza dois tipos de estudos: o processo de produção e avaliação do conhecimento científico em educação especial, por meio de estudos de metanálise e de abordagens bibliométricas e cientométricas da produção científica da área; e aqueles voltados para a produção de conhecimento diferenciado na área para fundamentar a formação de profissionais habilitados a avaliar, implantar, administrar e/ou orientar programas e serviços em educação especial.

\section{O conhecimento produzido na universidade impacta a prática da educação inclusiva fora dos muros acadêmicos?}

Um bom exemplo de como o conhecimento produzido em educação especial na universidade pode ter impacto fora dos muros acadêmicos são as oportunidades criadas por meio de programas de extensão ofertados à comunidade em geral. 
Com base em uma reflexão sobre a prática extensionista no campo da educação especial, realizada por docentes da UFSCar que atuaram no Programa de Formação Continuada de Professores na Educação Especial promovido pela Secretaria de Educação Especial do Ministério da Educação, nos anos de 2008 e 2009, pode-se ter uma dimensão aproximada desse impacto (MEC/SEESP, 2007).

Tal Programa teve por objetivo formar professores dos sistemas estaduais e municipais de ensino, por meio da constituição de uma rede nacional de instituições públicas de educação superior para ofertar cursos de formação continuada de professores na modalidade a distância. Nos dois editais, de 2008 e 2009, foram aprovados e oferecidos dois cursos de aperfeiçoamento, coordenados por docentes do PPGEEs/UFSCar: "Inclusão educacional do deficiente visual: baixa visão e cegueira na educação infantil" e "Formação de professores para a inclusão escolar do aluno com necessidades especiais". Cada curso foi oferecido a professores da rede pública de 20 municípios pertencentes a diversos estados brasileiros, totalizando cada uma das ofertas 500 alunos. Assim, no ano de 2008, foram atendidos 40 municípios, que inscreveram 1.000 professores, tendo a mesma situação ocorrido em 2009.

Outro aspecto a ser destacado diz respeito à modalidade do ensino a distância, que se constituiu em uma alternativa para a capacitação desses professores, os quais, na maioria das vezes, estão distantes geograficamente dos centros de produção do conhecimento científico.

Em termos da qualidade e profundidade dos conteúdos oferecidos, estes foram de alto nível, não só em virtude da formação e capacitação dos coordenadores dos cursos e docentes que prepararam o conteúdo das disciplinas ministradas, mas principalmente por envolverem alunos de Pós-Graduação em Educação Especial nas atividades de tutoria e preparação dos materiais oferecidos nas aulas. Para uma população-alvo tão carente de capacitação, esses cursos vieram preencher uma lacuna na formação desses profissionais que atuam nas salas de aula comum e no atendimento educacional especializado e que são responsáveis pela inclusão dos alunos. 


\section{Como as pesquisas podem transformar a realidade da educação especial encontrada hoje no Brasil?}

Um dos desafios que os pesquisadores enfrentam, após a geração do conhecimento científico, está relacionado com a aplicação e a divulgação do que foi produzido. A produção científica de teses e dissertações em educação especial, oriunda do PPGEEs/UFSCar, é um bom exemplo para mostrar como os trabalhos acadêmicos podem ultrapassar os muros da Universidade e auxiliar a transformar a realidade da educação especial encontrada no País. Podem ser citados, por exemplo, diversos estudos voltados para a construção e avaliação de programas de intervenção, seja de produtos ou de serviços, voltados para inserção de pessoas com deficiência na comunidade de maneira plena.

Alguns desses estudos referem-se a propostas de intervenção dirigidas a pais e familiares responsáveis por crianças com dificuldades de aprendizagem, incluídas nos primeiros anos do Ensino Fundamental de escola pública regular e que eram atendidas pelo serviço itinerante de educação especial (ROLFSEN, 2008); programa de intervenção sobre comunicação alternativa/ampliada (LIMA, 2008); programa de orientação para professores de alunos surdos incluídos (BOSCOLO, 2008); programas de intervenção para avaliação de bebês pré-termos e suas famílias, visando a oferecer subsídios para a prevenção de deficiências (FORMIGA, 2003); programa de ensino informatizado e individualizado para habilitar surdos no emprego do alfabeto digital (CARVALHO, 2005); programa de atividades recreativas para aprendizagem de leitura e escrita (LIMA, 2009); programa de atividade de construção de bonecos para crianças vítimas de violência com dificuldades de aprendizagem (SILVA, 2006); programa de habilidades sociais em situação natural de trabalho de pessoas com deficiência (CAMPOS, 2006); programa educacional de pessoas com autismo e deficiências severas (ASSUNÇÃO, 2004); programas educativos sobre o desenvolvimento infantil (PEREZ, 2003; NEÓFITI, 2009); programa de capacitação de docentes para pro- 
mover independência de crianças com baixa visão nas atividades de vida diária (GEBRAEL, 2009).

Por sua vez, a atividade de divulgação científica, entre os pesquisadores e cientistas, tem sido cada vez mais requisitada, a despeito do fato de que estes, após receberem os recursos das agências de fomento e realizarem a pesquisa, oferecem sua contrapartida não só para quem financiou suas pesquisas - por meio de relatórios científicos e técnicos mas também à comunidade a que pertencem, ao publicarem artigos, realizarem palestras e conferências, além de participarem de eventos científicos. Com isso, cumprem o ciclo de comunicação científica, desde a produção até divulgação do conhecimento científico. No entanto, em que pese essa divulgação ainda ser dirigida, na maioria das vezes, aos próprios pares, alguns esforços por parte dos cientistas já podem ser notados no sentido de fazer os resultados de suas pesquisas chegarem ao grande público, ou seja, a sociedade. Isso tem acontecido, por exemplo, por meio da participação de pesquisadores e cientistas em programas de popularização científica.

No caso específico dos pesquisadores da educação especial, a divulgação do conhecimento científico produzido no País tem sido promovida em eventos nacionais e regionais - tais como o Congresso Brasileiro de Educação Especial, o Congresso Brasileiro Multidisciplinar em Educação Especial, promovidos respectivamente pela UFSCar e pela UEL - os quais têm congregado grande número de participantes, o que cresce a cada ano, e que se constituem em foros privilegiados de troca de experiências e possibilidades de parcerias, visando ao aprofundamento de questões que afetam a educação especial na perspectiva da inclusão.

No entanto, é válido mencionar, como o fez Mendes (2008, p. 19) ao refletir sobre a produção de conhecimento na área de educação especial, que "pesquisar muito já não basta, é preciso produzir conhecimento novo. Também não basta criticar: é preciso saber perguntar e responder às demandas de conhecimento que não são poucas". 


\section{Há incentivos públicos ou privados de fomento à produção do conhecimento em educação especial no Brasil?}

Os exemplos citados anteriormente também podem ser ilustrativos dos incentivos públicos e privados de fomento à produção de conhecimento em educação especial no Brasil.

As agências de fomento à pesquisa científica, federais e estaduais, têm políticas de destinação de recursos, por meio de bolsas de iniciação científica, mestrado, doutorado e pós-doutorado no país e no exterior, além de apoio a projetos de pesquisa - vide, por exemplo, os editais anuais como o Universal e o de Ciências Humanas e Sociais do CNPq - que atendem parte da demanda de recursos para as pesquisas na área.

A já citada Secretaria de Educação Especial do Ministério da Educação, também tem oferecido um conjunto bastante amplo de publicações de cunho teórico e didático-pedagógico, fruto de seus programas e ações no campo da educação especial, nas quais colaboram pesquisadores e especialistas da área.

Outro programa apoiado pela Capes na área de educação especial é o Programa PROESP, uma parceria da Capes com a Secretaria de Educação Especial (Seesp/MEC), em que participam instituições de ensino superior e demais instituições que realizam projetos de pesquisa no âmbito da pós-graduação stricto sensu, nas diversas áreas do conhecimento envolvendo os estudos que tratem da educação especial, de acordo com o Programa Nacional de Apoio a Áreas Estratégicas (Pronap). Entre os objetivos do PROESP estão a formação e a pesquisa na área da educação especial sob a perspectiva da educação inclusiva, não substitutiva à educação regular, e as áreas temáticas do Programa focam a educação das pessoas com deficiência, transtornos globais do desenvolvimento e altas habilidades/superdotação.

Ao lado disso, em termos de apoio à divulgação científica consolidada em periódicos científicos e em trabalhos apresentados em eventos, a área de educação especial tem recebido recursos da Capes e de outras agências, por meio do Programa de Apoio a Publicações Periódicas e 
Programa de Apoio a Eventos. Como se vê, o apoio de agências públicas para a área de educação especial tem sido constante, mas insuficiente em termos de volume de recursos destinados e demanda solicitada. Por essa razão, esse apoio poderia ser intensificado, por meio de editais específicos de indução de pesquisas na área, a exemplo do que já ocorre em outras áreas de conhecimento.

No que diz respeito aos incentivos privados para fomento da produção do conhecimento em educação especial, salvo melhor juízo, estes ainda são inexistentes. O que se vê são iniciativas de caráter social, oriundas de empresas privadas, por meio de investimentos em "ações sociais" no contexto das chamadas "responsabilidades sociais das empresas" ou da "cidadania empresarial", como, por exemplo, a reserva de vagas para pessoas com deficiência em empregos.

No entanto, a maioria dessas iniciativas ocorre por força de legislação, sem uma preocupação maior com a capacitação, o treinamento e a absorção dessas pessoas no mercado de trabalho. No que se refere aos investimentos privados para a produção de conhecimento científico em educação especial, essa é uma área ainda a ser desbravada, a exemplo do que já ocorre, por exemplo, em outros campos de conhecimento, por meio de iniciativas de parcerias da pesquisa acadêmica com o setor produtivo.

\section{Conclusões}

Esse panorama sobre as pesquisas em educação especial no País, traçado com base em investigações que tiveram como foco a análise da produção científica da área, não teve a pretensão de ser exaustivo, mas tão somente pretendeu oferecer elementos a respeito dessa área que não é tão nova assim no País, mas cujos pesquisadores têm se dedicado a produzir conhecimento qualificado e cujos resultados, em sua maior parte, podem ser traduzidos em ações e intervenções junto à população-alvo dos estudos: as pessoas com deficiência. Ao mesmo tempo, é lícito mencionar que esse panorama apresentado não se esgota aqui, é apenas um ponto de 
partida para que o significado e alcance das pesquisas realizadas possam ser complementados com novas abordagens e outros olhares.

\section{Referências}

ALMEIDA, M. A.; MARQUEZINE, M. C. Produção científica do curso de especialização em deficiência mental da UEL: 1987-1997. Londrina: EDUEL, 1997. ASSUNÇÃO, K. R. Envolvimento de cuidadores no programa educacional de pessoas com autismo e deficiências severas: o que é e como medi-lo? 2004. 104 f. Dissertação (Mestrado em Educação Especial) - Universidade Federal de São Carlos, São Carlos, 2004.

BOSCOLO, C. C. Aplicação e avaliação de um programa de orientação para professores de alunos surdos incluídos. 2008. $131 \mathrm{f}$. Tese (Doutorado em Educação Especial) - Universidade Federal de São Carlos, São Carlos, 2008.

CAMPOS, J. A. de P. P. Programa de habilidades sociais em situação natural de trabalho de pessoas com deficiência: análise dos efeitos. 2006. $164 \mathrm{f}$. Tese (Doutorado em Educação Especial) - Universidade Federal de São Carlos, São Carlos, 2006.

CARVALHO, D. de. Programa de ensino informatizado e individualizado do alfabeto digital por equivalência de estímulos. 2005. 180 f. Dissertação (Mestrado em Educação Especial) - Universidade Federal de São Carlos, São Carlos, 2005.

FERREIRA, J. R. Pesquisa no contexto da política em educação especial. In: SEMINÁRIO BRASILEIRO DE PESQUISA EM EDUCAÇÃO ESPECIAL, 2., 1991, Rio de Janeiro. Anais... Rio de Janeiro: UERJ, 1991. p. 8-11.

FORMIGA, C. K. M. R. Programa de intervenção com bebês pré-termo e suas famílias: avaliação e subsídios para prevenção de deficiências. 2003. 238 f. Dissertação (Mestrado em Educação Especial) - Universidade Federal de São Carlos, São Carlos, 2003. 
GEBRAEL, T. L. R. Programa de capacitação de docentes para promover independência de crianças com baixa visão nas atividades de vida diária: PRÓ-AVD. 2009. 130 f. Dissertação (Mestrado em Educação Especial) - Universidade Federal de São Carlos, São Carlos, 2009.

LAPLANE, A. L. F.; LACERDA, C. B. F.; KASSAR, M. C. M. Abordagem qualitativa de pesquisa em educação especial: contribuições da etnografia. In: REUNIÃO DA ASSOCIAÇÃO NACIONAL DE PÓS-GRADUAÇÃO E PESQUISA EM EDUCAÇÃO, 29., 2006, Caxambu, MG. Anais... Rio de Janeiro: Anped, 2006.

LIMA, C. da S. E. Eficácia de um programa de comunicação alternativa aplicado a grupos de escolares com deficiência intelectual. 2008. 228 f. Dissertação (Mestrado em Educação Especial) - Universidade Federal de São Carlos, São Carlos, 2008.

LIMA, D. C. Programa de atividades recreativas para aprendizagem de leitura e escrita: contextualização das palavras ensinadas. 2009. $211 \mathrm{f}$. Dissertação (Mestrado em Educação Especial) - Universidade Federal de São Carlos, São Carlos, 2009.

LOURENÇO, E. A. G.; HAYASHI, M. C. P. I.; ALMEIDA, M. A. Delineamentos intrassujeitos nas dissertações e teses do PPGEEs/UFSCar. Revista Brasileira de Educação Especial, v. 15, n. 2, p. 319-336, 2009.

MARQUES, L. P. et al. Analisando as pesquisas em educação especial no Brasil. Revista Brasileira de Educação Especial, v. 14, n. 2, p. 251-272, 2008.

MAZZOTTA, M. S. Educação especial no Brasil: história e políticas públicas. São Paulo: Cortez, 1996.

MENDES, E. G. Deficiência mental: a construção científica de um conceito e a realidade educacional. 1995. 240 f. Tese (Doutorado em Psicologia Experimental) Instituto de Psicologia, Universidade de São Paulo, São Paulo, 1995.

MENDES, E. G. Pesquisas sobre inclusão escolar: revisão da agenda de um grupo de pesquisa. Revista Eletrônica de Educação, v. 2, n. 1, p. 3-25, 2008. 
MINISTÉRIO DA EDUCAÇÃO. SECRETARIA DE EDUCAÇÃO ESPECIAL. Brasília. Programa de Formação Continuada de Professores na Educação Especial. Disponível em: <http://portal.mec.gov.br/arquivos/pdf/2007_formacao.pdf〉. Acesso em: 12 maio 2010.

NEÓFITI, C. C. Educação para a vigilância do desenvolvimento infantil: formação virtual e presencial para educadores de creche. 2009. 208 f. Dissertação (Mestrado em Educação Especial) - Universidade Federal de São Carlos, São Carlos, 2009.

NUNES, L. R. O. P.; FERREIRA, J. R.; MENDES, E. G. A produção discente da pós-graduação em Educação e Psicologia sobre os indivíduos com necessidades educacionais especiais. In: MENDES, E. G.; ALMEIDA, M. A.; WILLIAMS, L. C. A. Temas em Educação Especial: avanços recentes. São Carlos: Ed. da UFSCar, 2004. p. 131-142.

PEREZ, R. M. Programa educativo sobre o desenvolvimento infantil para mães sociais. 2003. 132 f. Dissertação (Mestrado em Educação Especial) Universidade Federal de São Carlos, São Carlos, 2003.

PROGRAMA DE PÓS-GRADUAÇÃO EM EDUCAÇÃO ESPECIAL DA UFSCar. Disponível em: <http://www.ufscar.br/ cech/ppgees.html〉. Acesso em: 12 maio 2010.

ROLFSEN, A. B. Elaboração e avaliação de um programa de intervenção psicopedagógico para orientação de pais de crianças com dificuldades de aprendizagem -PPOP. 2008. 166 f. Dissertação (Mestrado em Educação Especial) - Universidade Federal de São Carlos, São Carlos, 2008.

SACARDO, M. Publicação científica derivada das dissertações e teses na interface entre Educação Física e Educação Especial. 2006. 134 f. Dissertação (Mestrado em Educação Especial) - Universidade Federal de São Carlos, São Carlos, 2006.

SÁNCHEZ GAMBOA, S. A. Fundamentos para la investigácion educativa: presupuestos epistemológicos que orientam al investigador. Santa Fé de Bogotá: Cooperativa Editorial Magisterio, 1998. 
SASSAKI, R. Terminologia sobre deficiência na era da inclusão. In: VIVARTA, V. (Org.). Mídia e deficiência. Brasília: Andi/FBB, 2003. p. 160-165. Disponível em: <http://www.andi.org.br/_pdfs/Midia_e_deficiencia.pdf >.Acesso em: 16 jan. 2010. SILVA, J. T. Elaboração, implementação e avaliação de um programa de atividade de construção de bonecos para crianças vítimas de violência com dificuldades de aprendizagem. 2006. 202 f. Dissertação (Mestrado em Educação Especial) - Universidade Federal de São Carlos, 2006.

SILVA, M. R. Análise bibliométrica da produção científica docente do Programa de Pós Graduação em Educação Especial da UFSCar. 2004. 177 f. Dissertação (Mestrado em Educação Especial) - Universidade Federal de São Carlos, São Carlos, 2004.

SILVA, R. H. R. Análise epistemológica das dissertações e teses defendidas no Programa de Pós Graduação em Educação Especial da UFSCar: 19812002. 2004. 279 f. Dissertação (Mestrado em Educação Especial) - Universidade Federal de São Carlos, São Carlos, 2004.

TORESAN, A.; REILY, L.; CAIADO, K. Panorama sobre a produção de conhecimento na área da deficiência nos Programas de Pós-Graduação do Estado de São Paulo. In: REUNIÃO ANUAL DA ASSOCIAÇÃO NACIONAL DE PÓS-GRADUAÇÃO E PESQUISA EM ADMINISTRAÇÃO, 18., 1995, Caxambu, MG. Anais... Caxambu, MG: ANPED, 1995.

Recebido: 09/06/2010

Received: 06/09/2009

Aprovado: 03/09/2010

Approved: 09/03/2010 